\title{
Tracheoscopic ventilation tube: a new step towards safer tracheostomy?
}

\author{
Benoit Voisin $^{1} \cdot$ Saad Nseir ${ }^{1,2}$
}

Received: 12 May 2016/Accepted: 12 May 2016/Published online: 24 June 2016

(C) Springer Science+Business Media Dordrecht 2016

Tracheostomy is an invasive procedure commonly performed in critically ill patients requiring mechanical ventilation for a long period of time. Based on the results of the largest epidemiological multinational study on mechanical ventilation, $14 \%$ of the 18,302 included patients required a tracheostomy during their stay in the intensive care unit [1]. Two recent meta-analyses found no significant impact of early compared with late tracheostomy on duration of mechanical ventilation, length of ICU stay, or mortality rate $[2,3]$. However, another recent meta-analysis found a possible reduction in pneumonia rate, when tracheostomy is performed early [4]. Further, other studies reported improved patient comfort and quality of life with tracheostomy, compared with endotracheal tube [5].

Since its first description by Ciaglia et al. [6], percutaneous dilatational tracheostomy (PDT) has become the most frequent technique for tracheostomy insertion, as reported by Vargas et al. [7]. Indeed, $75 \%$ of tracheostomy insertions are realized using the percutaneous method, including more than one half using PDT [7]. Nowadays, in the intensive care unit (ICU), the typical situation is PDT performed at bedside by ICU physician [7]. The key points of this widespread use are ease and safety [8-10].

The ease allows performing tracheostomy insertion commonly in the ICU, quickly with a procedure length of about 10 min [10], by the ICU physicians with a known learning curve [11].

The safety mainly results of the ease: a shorter procedure length compared to the surgical method reduces the

Saad Nseir

s-nseir@chru-lille.fr

1 Centre de Réanimation, CHU Lille, 59000 Lille, France

2 Faculté de Médecine, Univ. Lille, 59000 Lille, France stoma infection risk $[8,9,12]$, while the risk for complications during the procedure is reduced. However, serious adverse events are still reported [13,14]. The high rate of difficulty in placement and malposition [7, 15] up to $20 \%$, is the source of major intraprocedural events like hemorrhage or airway complications. In order to increase the ease and the safety of this invasive procedure, PDT has been improved over the time.

The first step had been an optimization of the dilatator's shape and material. Then, the need to monitor the airway during the tracheostomy insertion led to add a fiberoptic bronchoscopy to the procedure. For years, bronchoscopy guidance has been used as a safety adjunct, helping with the selection of site puncture and guiding the real-time entrance of the needle into the trachea. Nevertheless, this method is responsible for ventilation impairment related to tidal volume decrease, desaturation, hypercarbia and risk of intracranial hypertension [16].

Recently, a double-lumen endotracheal tube, and the use of ultrasound were investigated to improve the safety of PDT in the critically ill patient. In a preliminary study, Vargas et al. [17] evaluated the feasibility, gas exchange, and airway pressure in ten patients who received PDT through a double-lumen endotracheal tube divided into an upper channel that allows passage of a fiberoptic bronchoscope and a lower channel that is exclusively dedicated to patient ventilation. They concluded that PDT with double-lumen endotracheal tube can be performed safely without difficulties. Furthermore, during PDT, the use of this tube resulted in more stable gas exchange, airway pressures, and ventilation than PDT with a conventional endotracheal tube. One major limitation of the use of such a device is the need to reintubate the patient with this specific endotracheal tube. 
Ultrasound has emerged as a potentially useful tool to guide PDT and reduce procedure-related complications. Cervical echography can help to identify the cervical vasculature and the most appropriate location for the tracheal puncture site and to guide needle insertion into the trachea, similar to the technique used in ultrasound-guided vascular puncture. Gobatto et al. [10] conducted a randomized controlled trial $(n=118)$ to assess the non-inferiority of a ultrasound-guided PDT, compared with bronchoscopy-guided PDT. Procedure failure occurred in one $(1.7 \%)$ patient in each group, confirming the noninferiority hypothesis. Procedure-related minor complications occurred in $20(33.3 \%)$ patients in the ultrasound group, and in $12(20.7 \%)$ patients in the bronchoscopy group $(P=0.122)$.

Recent technological advances brought new tools, which could to help us to continue towards safer tracheostomy. In this issue of the Journal, Umutoglu et al. [18] investigated the feasibility of monitoring the airway during the PDT procedure with an EtView tracheoscopic ventilation tube (EtView TVT). The EtView TVT is an endotracheal tube with a camera at its tip, for continuous visualization of intratracheal structures and endotracheal tube position. They enrolled 24 patients scheduled for PDT in their ICU, and randomized them to receive PDT with standard airway monitoring by fiberoptic bronchoscopy, or by EtView TVT. Their data show an improvement in airway management during PDT. Visualization and identification of relevant airway structures in any steps of the PDT procedure were similar in the two study groups. The decrease in minute ventilation $(51 \pm 4$ vs. $12 \pm 7 \%)$, in $\mathrm{PaO}_{2}$ from initial levels during ( $34 \pm 21$ vs. $5 \pm 7 \%)$ and after $(26 \pm 27$ vs. $2.8 \pm 16 \%)$ the procedure were significantly $(P<0.05)$ higher in fiberoptic bronchoscopy, compared with EtView group. The authors should be congratulated for having conducted such an interesting trial, with important potential implications. However, as acknowledged by the authors, some limitations preclude definite conclusions, such as the single center design, the small number of included patients, the absence of blinding, and evaluation of clinical benefit. Further, the use of the EtView device requires reintubation. A previous study identified reintubation as an independent risk factor for ventilator-associated pneumonia [19]. Whilst microaspiration of contaminated secretions during reintubation might explain the association between reintubation and VAP, no difference was made in that study between reintubation for weaning failure, accidental extubation, or planned reintubation. A recent study, performed in a single center, reported zero VAP rate after planned reintubation with a new tracheal tube, providing impressive results on VAP prevention [20].

Based on the current evidence, only fiberoptic bronchoscopy or ultrasound could be recommended in routine for PDT performance (Figure). Further large studies should evaluate the double-lumen endotracheal tube and the EtView device. A combination of two methods, such as fiberoptic bronchoscopy and ultrasound, or double-lumen endotracheal tube/EtView device and ultrasound could also improve the safety of this procedure. Thus, the results published by Umutoglu et al. are interesting, because EtView TVT could be included into a bundle of tools to increase safety. Indeed, as a matter of safety, the assessment of synergy between different tools is often more effective than the quest for the ultimate one, which is always disappointing. On the way towards a safer tracheostomy, step by step...

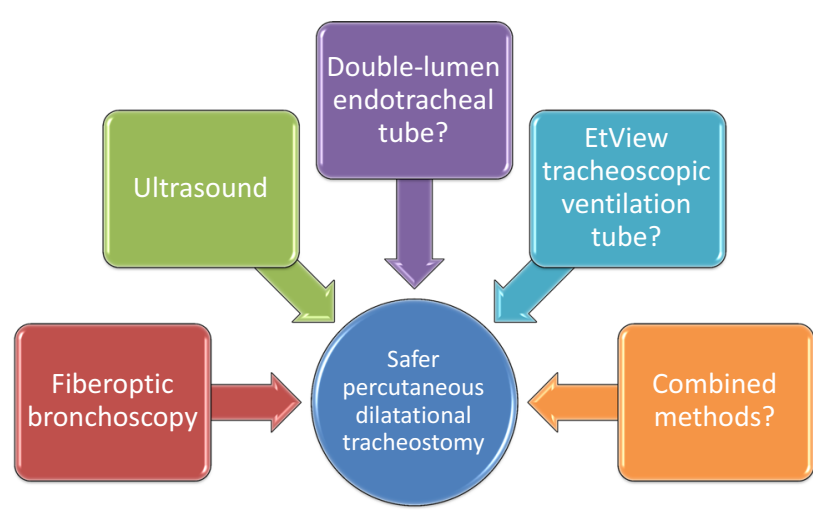

Conflicts of interest None.

\section{References}

1. Esteban A, Frutos-Vivar F, Muriel A, Ferguson ND, Peñuelas O, Abraira V, et al. Evolution of mortality over time in patients receiving mechanical ventilation. Am J Respir Crit Care Med. 2013;188(2):220-30.

2. Huang H, Li Y, Ariani F, Chen X, Lin J. Timing of tracheostomy in critically ill patients: a meta-analysis. PLoS One. 2014;9(3):e92981.

3. Szakmany T, Russell P, Wilkes AR, Hall JE. Effect of early tracheostomy on resource utilization and clinical outcomes in critically ill patients: meta-analysis of randomized controlled trials. Br J Anaesth. 2015;114(3):396-405.

4. Siempos II, Ntaidou TK, Filippidis FT, Choi AMK. Effect of early versus late or no tracheostomy on mortality and pneumonia of critically ill patients receiving mechanical ventilation: a systematic review and meta-analysis. Lancet Respir Med. 2015;3(2):150-8.

5. Freeman-Sanderson AL, Togher L, Elkins MR, Phipps PR. Return of voice for ventilated tracheostomy patients in ICU: a randomized controlled trial of early-targeted intervention. Crit Care Med. 2016;44(6):1075-81.

6. Ciaglia P, Firsching R, Syniec C. Elective percutaneous dilatational tracheostomy. a new simple bedside procedure; preliminary report. Chest. 1985;87(6):715-9.

7. Vargas M, Sutherasan Y, Antonelli M, Brunetti I, Corcione A, Laffey JG, et al. Tracheostomy procedures in the intensive care unit: an international survey. Crit Care. 2015 ;19(1):291. 
8. Delaney A, Bagshaw SM, Nalos M. Percutaneous dilatational tracheostomy versus surgical tracheostomy in critically ill patients: a systematic review and meta-analysis. Crit Care Lond Engl. 2006;10(2):R55.

9. Putensen C, Theuerkauf N, Guenther U, Vargas M, Pelosi P. Percutaneous and surgical tracheostomy in critically ill adult patients: a meta-analysis. Crit Care Lond Engl. 2014;18(6):544.

10. Gobatto ALN, Besen BAMP, Tierno PFGMM, Mendes PV, Cadamuro F, Joelsons D, et al. Ultrasound-guided percutaneous dilational tracheostomy versus bronchoscopy-guided percutaneous dilational tracheostomy in critically ill patients (TRACHUS): a randomized noninferiority controlled trial. Intensive Care Med. 2016;42(3):342-51.

11. Massick DD, Powell DM, Price PD, Chang SL, Squires G, Forrest LA, et al. Quantification of the learning curve for percutaneous dilatational tracheotomy. The Laryngoscope. 2000;110(2 Pt 1):222-8.

12. Dempsey GA, Morton B, Hammell C, Williams LT, Tudur Smith $\mathrm{C}$, Jones T. Long-term outcome following tracheostomy in critical care: a systematic review. Crit Care Med. 2016;44(3): 617-28.

13. Dennis BM, Eckert MJ, Gunter OL, Morris JA, May AK. Safety of bedside percutaneous tracheostomy in the critically ill: evaluation of more than 3,000 procedures. J Am Coll Surg. 2013;216(4):858-865-867.
14. Simon M, Metschke M, Braune SA, Püschel K, Kluge S. Death after percutaneous dilatational tracheostomy: a systematic review and analysis of risk factors. Crit Care Lond Engl. 2013;17(5):R258.

15. McCague A, Aljanabi H, Wong DT. Safety analysis of percutaneous dilational tracheostomies with bronchoscopy in the obese patient. The Laryngoscope. 2012;122(5):1031-4.

16. Ferraro F, Capasso A, Troise E, Lanza S, Azan G, Rispoli F, et al. Assessment of ventilation during the performance of elective endoscopic-guided percutaneous tracheostomy*: clinical evaluation of a new method. Chest. 2004;126(1):159-64.

17. Vargas M, Pelosi P, Tessitore G, Aloj F, Brunetti I, Arditi E, et al. Percutaneous dilatational tracheostomy with a double-lumen endotracheal tube. Chest. 2015;147(5):1267-74.

18. Umutoglu T, Bakan M, Topuz U, Yilmaz S, Idin K, Alver S, et al. Comparison of EtView ${ }^{\mathrm{TM}}$ tracheoscopic ventilation tube and video-assisted fiberoptic bronchoscopy during percutaneous dilatational tracheostomy. J Clin Monit Comput. 2016 (in press).

19. Torres A, Aznar R, Gatell JM, Jiménez P, González J, Ferrer A, et al. Incidence, risk, and prognosis factors of nosocomial pneumonia in mechanically ventilated patients. Am Rev Respir Dis. 1990;142(3):523-8.

20. Doyle A, Fletcher A, Carter J, Blunt M, Young P. The incidence of ventilator-associated pneumonia using the PneuX system with or without elective endotracheal tube exchange: a pilot study. BMC Res Notes. 2011;4:92. 